\title{
Speeding up the Hybrid-Monte-Carlo algorithm for dynamical fermions
}

M. Hasenbusch * and K. Jansen

DESY 01-155

NIC/DESY Zeuthen, Platanenallee 6, D-15735 Zeuthen, Germany

\begin{abstract}
We propose a modification of the Hybrid-Monte-Carlo algorithm that allows for a larger step-size of the integration scheme at constant acceptance rate. The key ingredient is the splitting of the pseudo-fermion action into two parts. We test our proposal at the example of the two-dimensional lattice Schwinger model and four-dimensional lattice QCD with two degenerate flavours of Wilson-fermions.
\end{abstract}

\section{INTRODUCTION}

In large scale simulations of lattice QCD with two flavours of mass-degenerate Wilson fermions, the mass of the fermions is still too large compared with the up and the down quark masses. Therefore a delicate extrapolation of the data is needed. Unfortunately, the numerical effort required for the Hybrid-Monte-Carlo (HMC) [1] algorithm increases as the quark mass decreases for, at least, three reasons [2, 3]: the solver (BiCGstab, conjugate gradient) needs more iterations, the step-size of the integration scheme has to be reduced to maintain a given acceptance rate, the autocorrelation times (in units of trajectories) increase.

We propose to modify the pseudo-fermion action in such a way that the problem of the decreasing step-size is drastically reduced. Our starting point is the observation that the stepsize can be increased when the fermion matrix is replaced by its preconditioned counter-part [4, 5 . This means that reducing the condition number of the fermion matrix allows for a larger step-size.

Here we shall present results for the twodimensional Schwinger model [6] and fourdimensional lattice QCD.

\section{THE SCHWINGER MODEL}

Let us start with the partition function with two degenerate flavours of Wilson-fermions

$Z=\int \mathrm{D}[U] \exp \left[-S_{G}(U)\right] \operatorname{det} M(U)^{\dagger} M(U)$

\footnotetext{
*presented by M. Hasenbusch
}

where $S_{G}(U)$ is the gauge action and $M(U)=$ $\mathbb{1}-\kappa H(U)$ the fermion matrix. In the HMC, the fermion determinant is represented by an integral over a so-called pseudo-fermion field $\phi$ :

$\operatorname{det} M^{\dagger} M \propto \int \mathrm{D}[\phi] \mathrm{D}\left[\phi^{\dagger}\right] \exp \left(-\left|M^{-1} \phi\right|^{2}\right)$.

Hence the pseudo-fermion action is given by $S_{F}=$ $\left|M^{-1} \phi\right|^{2}$. The key-ingredient of our method is to split $M$ into two factors, where each factor has a reduced condition number. For each factor we use a pseudo-fermion field:

$S_{F 1}=\left|\tilde{M}^{-1} \psi\right|^{2} \quad S_{F 2}=\left|\tilde{M} M^{-1} \phi\right|^{2} ，$

where $\tilde{M}=\mathbb{1}-\tilde{\kappa} H$ with $0 \leq \tilde{\kappa} \leq \kappa$.

In the HMC we have to compute the variation of the action with respect to the gauge-field. It turns out that for our modified action this can be done in much the same way as for the standard pseudo-fermion action:

$\delta S_{F 2}(U, \phi)=-\left[Y^{\dagger} \delta M X+X^{\dagger} \delta M^{\dagger} Y\right]$

with $X=M^{-1} \phi$ and $Y=M^{\dagger-1}(a \phi+b X)$, where $a=\tilde{\kappa} / \kappa$ and $b=1-a$.

In our numerical study of the Schwinger model, we have applied this modification on top of evenodd preconditioning: $M$ is replaced by $M_{e e}=$ $\mathbb{1}_{e e}-\kappa^{2} H_{e o} H_{o e}$. The modified pseudo-fermion action is given by

$S_{F 1}=\left|\tilde{M}_{e e}^{-1} \psi\right|^{2} \quad S_{F 2}=\left|\tilde{M}_{e e} M_{e e}^{-1} \phi\right|^{2}$,

where $\tilde{M}_{e e}=\mathbb{1}_{e e}-\tilde{\kappa}^{2} H_{e o} H_{o e}$. We have performed our simulations of the Schwinger model at the same parameters as ref. 何. We have used 


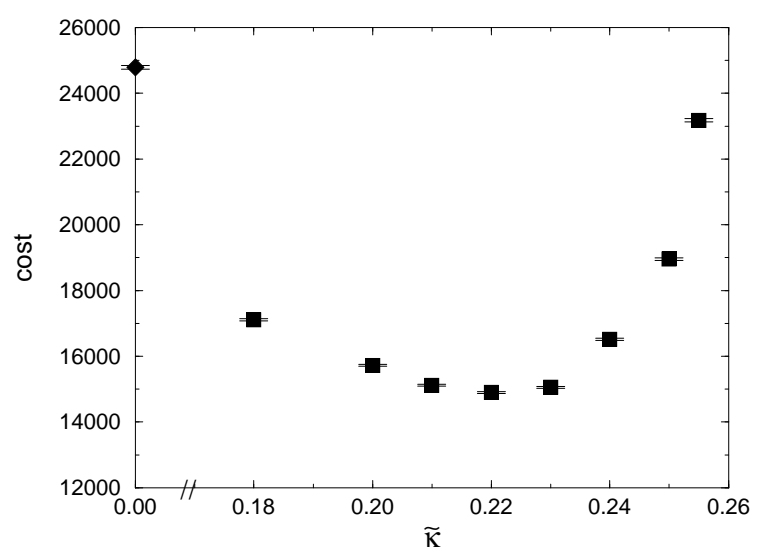

Figure 1. cost $=$ (number of applications of $H_{e o} H_{o e}$ per trajectory) as a function of $\tilde{\kappa}$ for $L=32, \beta=4.0$ and $\kappa=0.26$. The acceptance rate is fixed to $\approx 0.8$.

the leap-frog integration-scheme. In order to separate off effects from the gauge action, we used a reduced step-size $(n=4)$ for the gauge action [7]. The length of the trajectory is taken randomly between 0.5 and 1.5. We used the BiCGstab solver to compute $\tilde{M}_{e e}^{-1} \psi$ and $M_{e e}^{-1} \phi$.

In all cases we have chosen $\beta=4.0$. In a first set of runs we simulated a $32 \times 32$ lattice at $\kappa=0.26$ and various values of $\tilde{\kappa}$. Following ref. [4] the pseudo-meson mass is $m_{P}=0.210$ (3) for this value of $\kappa$. Each run consists of 10000 trajectories. 1000 trajectories were discarded for equilibration. We have measured the value of square Wilson-loops up to the size $5 \times 5$ and the topological charge with the geometrical definition. We found that for fixed acceptance rate, the integrated auto-correlation times of these quantities do not depend on the value of $\tilde{\kappa}$, within errorbars. Therefore, the performance of the algorithm can be measured just by the number of applications of $H_{e o} H_{o e}$ that is needed in average for one trajectory. This number is given as a function of $\tilde{\kappa}$ in figure 1. We see that the numerical cost has a shallow minimum at $\tilde{\kappa} \approx 0.22$. At this minimum cost is reduced by a factor of about 1.66 compared with the standard HMC $(\tilde{\kappa}=0)$.
Next we performed simulations of a $64 \times 64$ lattice at $\kappa=0.2570$ and $\kappa=0.2605$. The pseudo-meson masses at these values of $\kappa$ are $m_{P}=0.210(3)$ and $m_{P}=0.124(5)$, respectively 顿. For $\kappa=0.2605$ we found a reduction of the cost by factor of 2 compared with the standard pseudo-fermion action. For $\kappa=0.257$ we see only a tiny improvement. This observation indicates that the gain in performance increases as we approach $\kappa_{c}$. For details see ref. [6].

\section{LATTICE QCD}

We have performed some exploratory runs for QCD in four dimensions. Here we used a different set-up as in the case of the Schwinger model. These changes are mostly motivated by the existing HMC-code of the ALPHA-collaboration.

We considered the Hermitian fermion matrix $Q=c_{0} \gamma_{5} M$. We define $\tilde{Q}=Q+i \sigma \mathbb{1}$. That means that the modified pseudo-fermion action consists of the two parts

$S_{F 1}=\psi^{\dagger}\left[Q^{2}+\sigma^{2} \mathbb{1}\right]^{-1} \psi$,
$S_{F 2}=\phi^{\dagger}\left[\mathbb{1}+\sigma^{2}\left(Q^{2}\right)^{-1}\right] \phi$.

In our simulations we have used $O(a)$ clover improvement and even-odd preconditioning 8 .

Instead of the leap-frog scheme we used a scheme proposed in ref. [7] that partially removes the $\Delta \tau^{2}$ errors. This integration scheme is characterised by the coefficients $1 / 6,1 / 2,2 / 3$, $1 / 2$ and $1 / 6$. Note that in contrast to the leapfrog scheme, the derivative of the fermion action with respect to the gauge-field has to be computed twice per elementary step.

In a first set of runs we studied an $L^{4}$ system with Schrödinger-functional (SF) boundary conditions, where $\kappa \approx \kappa_{c}$. The parameter $\sigma$ of the modified action is set as $\sigma^{2}=\sqrt{\lambda_{\min }}$, where $\lambda_{\min }$ is the smallest eigenvalue of $\hat{Q}^{2}$. Our results are summarised in table 11. For $L=8$ the step-size is enlarged by a factor of 1.4 by the modification of the action, while the overhead is about 1.29. For $L=12$, we see already an increase of the step size by a factor of 2 , while the over-head is 1.28 .

Finally we performed runs at a smaller value of $\beta$ for a physically large volume. We took the 
Table 1

Runs with SF-boundary conditions. $\Delta \tau$ is the step-size of the integration-scheme, $N_{m d}$ the number of steps per trajectory, "acc" the acceptance rate. $N_{C G}$ and $N_{C G 2}$ are the average number of iterations needed to compute $\left(\hat{Q}^{2}\right)^{-1} \phi$ and $\left[\hat{Q}^{2}+\sigma^{2} \mathbb{1}\right]^{-1} \psi$ with the conjugate gradient solver. The standard HMC data are taken from ref. [5].

\begin{tabular}{rllrlrr}
\hline$L$ & $\beta$ & $\Delta \tau$ & $N_{m d}$ & acc & $N_{C G}$ & $N_{C G 2}$ \\
\hline 8 & 7.2103 & 0.14 & 7 & $92 \%$ & 79 & 23 \\
8 & 7.2103 & 0.10 & 10 & $92 \%$ & 80 & \\
\hline 12 & 7.5 & 0.14 & 7 & $84 \%$ & 114 & 32 \\
12 & 7.5 & 0.075 & 13 & $89 \%$ & 114 & \\
\hline
\end{tabular}

Table 2

Runs for $\beta=5.2, \kappa=0.1370$ and $c_{s w}=1.76$ with periodic space and anti-periodic time boundary conditions on a $8^{3} \times 24$ lattice.

\begin{tabular}{lllll}
\hline$\Delta \tau$ & $N_{m d}$ & acc & $N_{C G}$ & $N_{C G 2}$ \\
\hline 0.06 & 16 & $82 \%$ & 122 & \\
0.1 & 10 & $80 \%$ & 122 & 27 \\
\hline
\end{tabular}

parameters for the run from ref. [9]. In ref. [9] the leap-frog scheme was used. For $\Delta \tau=0.02$ and $N_{m d}=50$ elementary steps per trajectory they obtained an acceptance rate of $80 \%$.

Our results are given in table 2. For these particular parameters, the improved integration scheme performs better than the leap-frog. With the standard pseudo-fermion action we get an acceptance rate of $82 \%$ with $\Delta \tau=0.06$. I.e. the improved scheme out-performs the leap-frog by a factor of 1.5. Using the modified action, the stepsize can be further increased to 0.1 , maintaining an acceptance rate of $80 \%$.

\section{CONCLUSIONS}

We propose to use a modified pseudo-fermion action in the HMC simulation of dynamical Wilson-fermions. It is easy to incooperate this modification in an existing HMC Code. We have demonstrated that the modification reduces the numerical costs for the 2D Schwinger model as well as 4D QCD with clover-improvement. We see an improvement up to a factor of 2 . However, we expect that for lighter quark masses this gain becomes even larger.

There remain a number of open problems. We need longer runs for QCD to determine reliable autocorrelation times. We have to compare the modification of the pseudo-fermion action with $\tilde{M}$ and $\tilde{Q}$ in the same model. Is it useful to split the pseudo-fermion action in more than two parts? We have to study more carefully the inter-play of various integration schemes with the modification of the action.

Finally, our idea can be combined with Polynomial-Hybrid-Monte-Carlo algorithm 10 , 11]. (See Mike Peardons plenary talk). The implementation is straight forward. The non-trivial question is the choice of the polynomials.

\section{ACKNOWLEDGEMENT}

We like to thank R. Sommer for discussions.

\section{REFERENCES}

1. S. Duane, A.D. Kennedy, B.J. Pendleton and D. Roweth, Phys.Lett.B 195 (1987) 216.

2. R. Gupta, A. Patel, C.F. Baillie, G. Guralnik, G.W. Kilcup and S.R. Sharpe, Phys. Rev. D 40 (1989) 2072.

3. K. Jansen, Nucl.Phys.Proc.Suppl. 53 (1997) 127.

4. M.J. Peardon, hep-lat/0011080.

5. R. Frezzotti, M. Hasenbusch, J. Heitger, K. Jansen and U. Wolff, Comput. Phys. Commun. 136 (2001) 1.

6. M.Hasenbusch, hep-lat/0107019, to be published in Phys.Lett.B

7. J.C. Sexton and D.H. Weingarten, Nucl. Phys. B380 (1992) 665.

8. K. Jansen and C. Liu, Comput. Phys. Commun. 99 (1997) 221.

9. Z. Sroczynski, Ph.D. thesis, Edinburgh (1998).

10. P. de Forcrand and T. Takaishi, Nucl. Phys. Proc. Suppl. 53 (1997) 968.

11. R. Frezzotti and K. Jansen, Phys. Lett. B 402 (1997) 328. 